\title{
The Study on Color Design of the Interior Space in the High School
}

\author{
Library \\ Hui LIU ${ }^{1, a, *}$ and Fei-hu CHEN²,b \\ ${ }^{1}$ The Environmental Art Design Studio of School of Architecture of Hunan University, Yuelu District, \\ Changsha city, Hunan province, China. \\ ${ }^{2}$ The Environmental Art Design Studio of School of Architecture of Hunan University, Yuelu District, \\ Changsha city, Hunan province, China. \\ a962215348@qq.com, b188000786@qq.com \\ * Hui LIU
}

Keywords: The high school library,Interior space color, The utilization of color

Abstract.Color plays a significant role in the interior space design of high school libraries. From the perspective of the color's influence on library users, this study analyzes how the scientific use of color can provide readers with high-quality reading atmosphere and explores the new development trends of the library construction.

\section{Introduction}

The high school library is a second classroom where students absorb knowledge and develop learning interests. The first visual impression of the library for juveniles is color, and the next are shapes, material and other factors. The library used to be stiff and monotonous because of the economic and conceptual limitations, as a result, students lacked the consciousness of using the library, making negative effect on the working of the library. While a systematic color impression can contribute to forming the perceptual space of the library, and this will benefit the young readers' learning emotions, the process of thinking, psychological health and aesthetic conception. This color impression is also the source of the sense of belonging in the library. Color, an important aspect of the library environment, is an important cultural carrier for the library; therefore the study on interior space color of the high school library is of practical significance.

\section{The explanation of interior space color of the high school library}

\section{The concept of interior space color of the high school library}

Interior space color of the high school library, in a broad way, is the sum of all appreciable colors in the interior space of the whole library. In a narrow sense, it refers to the relatively comprehensive color vision of all appreciable objects in the library interior space.

The concept of interior space color design of the high school library

Interior space color design of the high school library aims at building a comfortable and beautiful environment with reasonable functions where students' physical and mental needs can be satisfied, through new materials, technology, and principles of color design, according to the nature of the usage of the library, relative standards and particular surroundings. This interior space design, based on the library's specific features, enables the library work as a practical building which meets the relative function requirements. Meanwhile, such spiritual elements as the style of the library, 
campus culture, and atmosphere will be reflected.

\section{The influence of the color in high school library interior space on readers}

The color design of high school library is vital, because the color collocation and decoration affect readers' physiological and psychological conditions.

Physiologists insist that men's sense organs turn physical color stimuli into nervous impulses and deliver them to the brain, and then a series of sensations and perceptions are produced. The green color, in the middle of spectrum, causes the least visual fatigue, which is called "physiological balanced color". If this kind of balanced color is absent in the library interior space, a visual balance and rest can not be reached, and reader's thinking can be affected even the comprehension and assimilation of knowledge will be reduced. So as for the interior space color design of the high school library, the color balance is supposed to be taken into consideration to create a harmonious and comfortable color environment, no matter the amount of color collocations is big or small, no matter the colors are similar or in contrast.

Besides the physiological influence, color also impacts readers' psychology. Men have different associations with different colors stemming from different life experiences. These associations can be direct and figurative, or indirect and abstract. But color's influence on men's psychology, in a certain way, shares similarities. The reactions to the strength, depth, expansion, temperature of color are not that different. The library should be designed elaborately and overall, from readers' psychological needs, according to the rule of color collocations and particular groups, constructing a comfortable, quiet, fresh and harmonious reading environment.

\section{The utilization of color in the high school library interior space design}

\section{The utilization principles of high school library interior space color}

The subsection heading of this paragraph is "color contrast". Usually different function zones are distinguished by different color schemes. The same function zones are in the same color scheme; while the zones which are demanded to be outstanding or beautified are in color contrasts; the transition zones are with transition colors. Color of the zones for reading and placing books tend to be warm, soft, and light; public zones' colors are bright and strong; and transition zones are better to be with neutral colors. Taking the library of An hua County First High School, whose design is directed by the first author, as an example, the public zone (fig. 1) is decorated by red; the reading zone(fig. 2 fig.3) are painted with green and yellow respectively; the soft burlywood color is adopted in the transition zone(fig.4), reaching an overall balance.

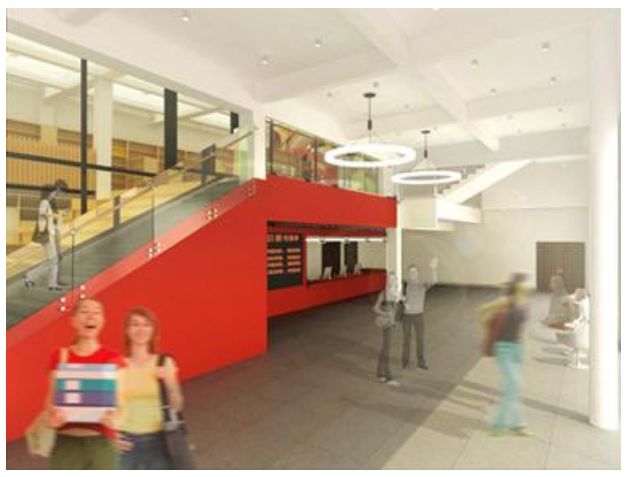

Fig. 1: Public Zone

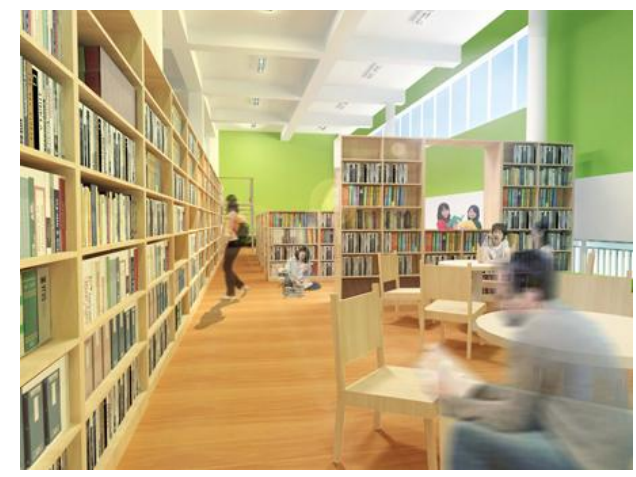

Fig. 2: Reading Zone 1 


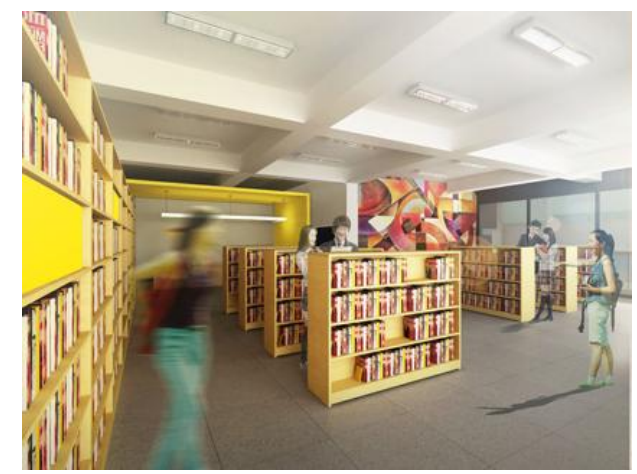

Fig. 3: Reading Zone 2

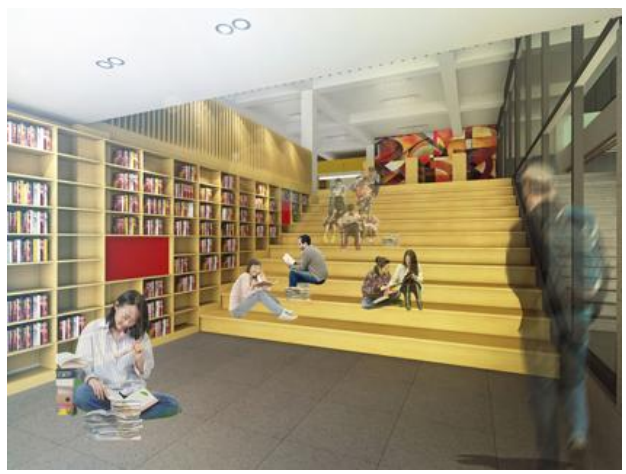

Fig. 4: Transition Zone

The subsection heading of this paragraph is "the harmony and balance of color". The harmony and balance of color depends on the area, location, purity, lightness, hue of color. The balance of high school library color is a state where readers' vision is balanced. In the application of bright colors, the purity, brightness, hue, and area of color must be controlled. Otherwise, a gaudy, complicated reading environment will cause emotional and thinking disorders in readers' minds. The Reading Zone 1 of the library of An hua County First High School (fig.5 fig.6) is ornamented with bright green, by a proper control of purity, lightness and color distribution areas. The walls in fig.5 are mainly painted light green and the dark green chairs and shelves are interspersed there. A color balance and gradation are produced in this zone.

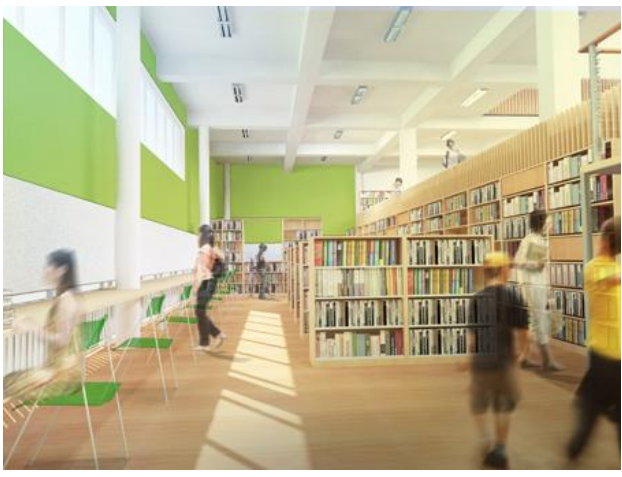

Fig. 5: Reading Zone 1

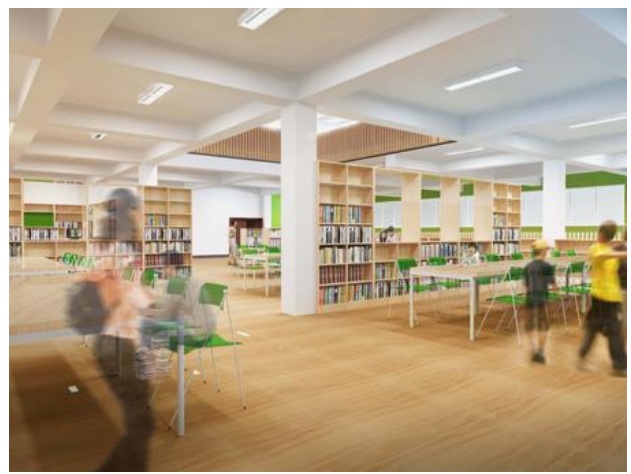

Fig. 6: Reading Zone 1

The subsection heading of this paragraph is "the special function of color". The green color, in the middle of spectrum, called "physiological balanced color", can partly eliminate such negative emotions as fatigue, bring equilibrium and tranquilize men. If some green plants are placed in the high school library, a light and comfortable visual sense can be made to lessen young readers' visual fatigue. Fig. 7 shows the Reading Zone 3 of the library of An hua County First High School. Green plants in this zone create a relaxing reading atmosphere and trigger reading impulses.
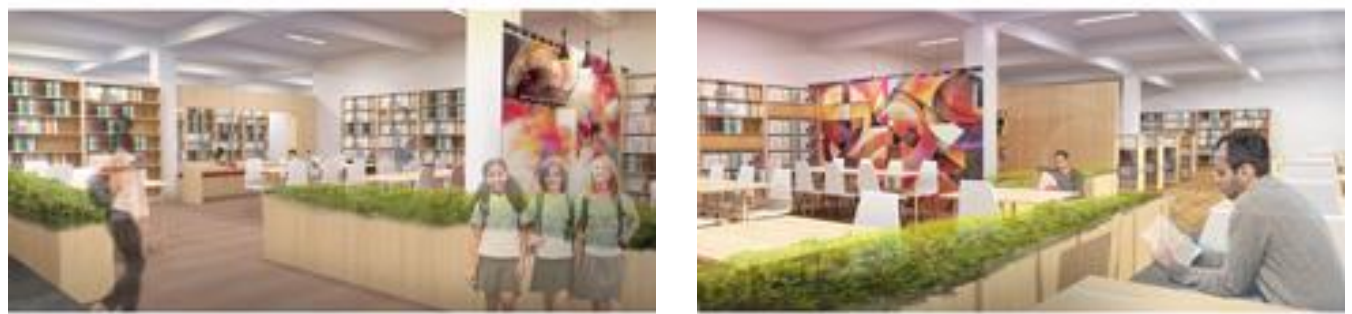

Fig. 7: Reading Zone 3 


\section{The enhancement of the application of color in the high school library interior space design}

Color carries rich meanings and symbols. Juveniles often have physiological reactions and psychological associations by natural feelings. Additionally, they are in a period when they entertain strong thirst for knowledge, so they are more sensitive to color and monotonous color can not satisfy the need the thirst of the teenagers with strong learning ability. Therefore, in the design of the high school library interior space, it is young readers' spiritual need to enhance the use of color. Then how to manage to do it? There are three aspects to follow:

The subsection heading of this paragraph is the diversity of color. Psychologists believe that the proper usage of color is conductive to adjusting men's emotions. The color of objects can leave juveniles more direct vision than the shape, which leads to directly affecting their psychology and emotion. Bright color is accordance with their lively psychological period when they are curious about their surroundings. In such library the color visual changes can arouse young readers learning interests and delightful mood. The design of the library of An hua County First High School is based on its functions and characters. Red, yellow, green, and white are used in the first floor (e.g. fig.1, fig.4), the second floor (e.g.fig.3), the third floor (e.g.fig2, fig5, fig6), and the fourth floor (e.g.fig.7) respectively. This design not only meets the particular spatial needs but also increases the variety of color in the library and motivates users' reading interests.

The subsection heading of this paragraph is "the saturability and contrast of color". In the high school library indoor environment, the visual vigor is from the proper saturability and contrast which ought to be controlled and balanced. Usually a soft color is used as the domain hue in the design of the library interior space, and strong saturated colors, like red, green, orange, are accent colors. This reading space fits lively and adorable children. The strong contrast of domain color and accent colors makes young readers feel the vigor and joy in the reading space, and they will spend more time being close to this pure, bright, and soft space and absorbing knowledge. In the library of An hua County First High School, with burlywood and white as dominant colors, different suited colors are interspersed in there, creating different reading emotions.

The subsection heading of this paragraph is "proper addition of warm color". The design of the library interior space color is not settled. It is generally widely believed that the warm color excites men and the cool color calms men down. But if there is an excess of cool colors in the library, students are likely to feel visually nervous and down, losing reading interests. So an appropriate area of warm color not only helps to keep the young readers focus and calm, but also contributes to promoting their thinking, understanding and assimilating of knowledge.

\section{The new trends of the high school library interior space color}

Color is a key factor of the design for a unique high school library meeting the psychological, sociological, mental, and material demands of a certain group. New concepts, new thoughts, new technology promote the emotional communication, humanization, cultural demonstration in the color design of the high school library interior space.

\section{Emotional communication}

The free art of color in the design of high school library interior space is not only to encourage the young readers to read but to help them to interact to the library environment. In a reading environment with vigor and joy, they will naturally make emotional communication with the surroundings.

\section{The humanized design}

The humanized design of the high school library interior space is to use the colors juveniles are interested in to create learning space, based on their thinking patterns, sociological needs, 
behavioral habits. Different activity zones are marked with different blocks of bright colors attracting users, who will experience a comfortable and gentle, scientific and convenient learning process.

\section{The demonstration of local cultures}

Every high school has different cultures due to different regions and histories. The high school libraries are cultural marks which are unique and reflecting different cultures and aesthetics. Color is an important part of the library interior space design. The color collocations are carrying both local historical culture and local aesthetics. Demonstrating different local cultures, the color design of the high school interior space absorbs new ideas, new techniques, new forms and new materials to build every specialty, combining regional characteristics and development demands.

\section{Conclusion}

If the color is well utilized to decorate the high school library following color collocations principles and color rules, the young readers can enjoy reading and learning in a comfortable, quiet, fresh and harmonious library and have the sense of belonging. Furthermore, their thinking, aesthetic conception, and learning efficiency will be improved. Hence, the color design of the high school interior space can bring significant benefits to the high school library construction.

\section{References}

[1] Yuanxin $\mathrm{Wu}$, The psychological effect of color and its utilization in design: submitted to Fujian building, In Chinese, (2005(5): 4-5).

[2] Minghua Yang, The explanation of architectural design specialty of Shantou University library: submitted to Library Tribune, In Chinese ,(2010(1):62-63).

[3] Minghong Chen, The study of library color collocations: submitted to Library Development, In Chinese, (1997(3)) .

[4] Lingxin Kong, The color culture of the library interior space: submitted to Theory and Practice of Higher Education in Building Material Science, ,In Chinese, (1995(4)) .

[5] Sha Liu, Color's cultural function in modern libraries: submitted to Shang Dong Library Quarterly, In Chinese, (2004(3)).

[6] Mingzhe Hu, Color expression—subjective color schemes skills, Bei Jing, In Chinese, 2005. 